\title{
Does 3D-Assisted Operative Treatment of Pelvic Ring Injuries Improve Patient Outcome?-A Systematic Review of the Literature
}

\author{
Hester Banierink ${ }^{1, *}$, Anne M. L. Meesters ${ }^{1}{ }^{(\mathbb{D}}$, Kaj ten Duis ${ }^{1}$, Job N. Doornberg ${ }^{1,2}$, Mostafa El Moumni ${ }^{1}$, \\ Erik Heineman ${ }^{3}$, Inge H. F. Reininga ${ }^{1}$ and Frank F. A. IJpma ${ }^{1}$ \\ 1 Department of Trauma Surgery, University Medical Center Groningen, University of Groningen, \\ 9713 GZ Groningen, The Netherlands; a.m.l.meesters@umcg.nl (A.M.L.M.); k.ten.duis@umcg.nl (K.t.D.); \\ j.n.doornberg@umcg.nl (J.N.D.); m.el.moumni@umcg.nl (M.E.M.); i.h.f.reininga@umcg.nl (I.H.F.R.); \\ f.f.a.ijpma@umcg.nl (F.F.A.I.) \\ 2 Department of Orthopedics, University Medical Center Groningen, University of Groningen, \\ 9713 GZ Groningen, The Netherlands \\ 3 Department of Surgery, University Medical Center Groningen, University of Groningen, \\ 9713 GZ Groningen, The Netherlands; e.heineman@umcg.nl \\ * Correspondence: h.banierink@umcg.nl; Tel.: +31-(0)642-665-618; Fax: +31-(050)361-4588
}

Citation: Banierink, H.; Meesters, A.M.L.; ten Duis, K.; Doornberg, J.N.; El Moumni, M.; Heineman, E.; Reininga, I.H.F.; IJpma, F.F.A. Does 3D-Assisted Operative Treatment of Pelvic Ring Injuries Improve Patient Outcome?-A Systematic Review of the Literature. J. Pers. Med. 2021, 11, 930. https://doi.org/10.3390/ jpm11090930

Academic Editor: Pierluigi Maria Rinaldi

Received: 26 July 2021

Accepted: 17 September 2021

Published: 18 September 2021

Publisher's Note: MDPI stays neutral with regard to jurisdictional claims in published maps and institutional affiliations.

Copyright: (c) 2021 by the authors. Licensee MDPI, Basel, Switzerland. This article is an open access article distributed under the terms and conditions of the Creative Commons Attribution (CC BY) license (https:/ / creativecommons.org/licenses/by/ $4.0 /)$.

\begin{abstract}
Background: There has been an exponential growth in the use of advanced technologies for three-dimensional (3D) virtual pre- and intra-operative planning of pelvic ring injury surgery but potential benefits remain unclear. The purpose of this study was to evaluate differences in intra- and post-operative results between 3D and conventional (2D) surgery. Methods: A systematic review was performed including published studies between 1 January 2010 and 22 May 2020 on all available $3 \mathrm{D}$ techniques in pelvic ring injury surgery. Studies were assessed for their methodological quality according to the Modified McMaster Critical Review form. Differences in operation time, blood loss, fluoroscopy time, screw malposition rate, fracture reduction and functional outcome between 3D-assisted and conventional (2D) pelvic injury treatment were evaluated and a best-evidence synthesis was performed. Results: Eighteen studies fulfilled the inclusion criteria, evaluating a total of 988 patients. Overall quality was moderate. Regarding intra-operative results of 3D-assisted versus conventional surgery: The weighted mean operation time per screw was 43 min versus $52 \mathrm{~min}$; for overall operation time $126 \mathrm{~min}$ versus $141 \mathrm{~min}$; blood loss $275 \pm 197 \mathrm{~mL}$ versus $549 \pm 404 \mathrm{~mL}$; fluoroscopy time $74 \mathrm{~s}$ versus $125 \mathrm{~s}$ and fluoroscopy frequency $29 \pm 4$ versus $63 \pm 3$. In terms of postoperative outcomes of 3D-assisted versus conventional surgery: weighted mean screw malposition rate was $8 \%$ versus $18 \%$; quality of fracture reduction measured by the total excellent/good rate by Matta was $86 \%$ versus $82 \%$ and Majeed excellent/good rate $88 \%$ versus $83 \%$. Conclusion: The 3Dassisted surgery technologies seem to have a positive effect on operation time, blood loss, fluoroscopy dose, time and frequency as well as accuracy of screw placement. No improvement in clinical outcome in terms of fracture reduction and functional outcome has been established so far. Due to a wide range of methodological quality and heterogeneity between the included studies, results should be interpreted with caution.
\end{abstract}

Keywords: pelvic ring injury; sacroiliac screw; three-dimensional; 3D virtual surgical planning; 3D printing; navigation

\section{Introduction}

Pelvic ring injuries have an estimated annual incidence of $14-37$ per 100,000 inhabitants each year [1,2]. Treatment can be either non-operative or operative, depending on the injury as well as patient characteristics. The operative treatment of pelvic ring injuries remains a challenging task for surgeons due to the complex three-dimensional (3D) shape of the pelvis, morphological variations, limited access to fracture sites, and narrow bone 
corridors for screw placement [3]. The goal of operative treatment is to restore pelvic symmetry and achieve stable fracture fixation, which allows for early mobilization and good functional outcome at the long-term $[4,5]$. Progress in 3D imaging technologies has resulted in an exponential increase in the usage of these techniques-that is both industry- as well as surgeon-driven- for preoperative planning and for translation of the plan to the operative procedure [6]. In essence, 3D-assisted surgery encompasses a wide spectrum of modalities including 3D virtual preoperative planning, 3D-printed models for pre-contouring of osteosynthesis plates and 3D navigational tools. Some coin these 3D (printing) techniques the "second industrial revolution" in Orthopaedic Trauma Surgery. Nevertheless, the additional clinical value of 3D techniques in pelvic surgery has yet to be elucidated, both practically as well as scientifically.

Conventional X-rays and two-dimensional (2D) computed tomography (CT) images are to date widely used to assess fracture characteristics, reduction quality and positions of osteosynthesis materials in pelvic ring injury treatment [3]. However, $3 \mathrm{D}$ virtual models may allow the surgeon to gain more insight in the fracture pattern, surgical approach, and positions of osteosynthesis materials. It has been reported that pre-operative virtual simulation and 3D printing-assisted pre-contoured plate fixation of pelvic ring injuries resulted in precise pre-operative planning and accurate execution of the operative procedures [3]. Moreover, 3D-assisted surgery for percutaneous screw placement may lower the risk of complications and decrease the need for revision surgery due to a lower rate of screw malposition [7]. However, there is a lack of studies with sufficient statistical power to provide evidence on superiority of the available 3D technologies compared to conventional (2D) techniques in different types of pelvic ring injuries.

Hence, the main objective of the present systematic review was to analyse differences in outcomes between currently available 3D-assisted and conventional (2D) pelvic ring injury treatment. Therefore, we asked (1) What is the difference in intra-operative results in terms of operation time, blood loss, screw malposition and fluoroscopy time between 3D-assisted and conventional (2D) surgery? and (2) What is the difference in post-operative results in terms of fracture reduction and functional outcome between $3 \mathrm{D}$ assisted and conventional (2D) surgery?

\section{Methods}

This systematic review was performed according to the Preferred Reporting Items for Systematic Reviews (PRISMA) [8]. The review protocol has been registered in PROSPERO International prospective register of systematic reviews under registration number CRD42021224915.

\subsection{Identification of Studies: Search Strategy}

The MEDLINE-Pubmed and Ovid-EMBASE libraries were searched on May 22nd of 2020 for articles published between 1 January 2010 until 22 May 2020. The search string was developed in collaboration with an experienced medical librarian (Table 1). It was developed to identify references related to 3D-imaging and 3D-operative techniques of pelvic ring injuries. Therefore, the items "pelvis", "injury" and "3D/threedimension" were combined to develop the search strategy.

Table 1. Search strings by database.

\begin{tabular}{|c|c|}
\hline Database & Search String \\
\hline MEDLINE-PubMed & $\begin{array}{c}\text { ((("Pelvis"[Mesh] OR pelvic ring[tiab]) AND ("Wounds and Injuries"[Mesh] OR "injuries" } \\
\text { [Subheading] OR injur*[tiab] OR fractur*[tiab]))) AND ((3D[tiab] OR three dimension*[tiab] OR } \\
3 \text { dimension*[tiab] OR "Printing, Three-Dimensional"[Mesh] OR "Imaging, Three-Dimensional”[Mesh] }\end{array}$ \\
\hline Ovid-EMBASE & $\begin{array}{l}\text { ('pelvis' / exp OR 'pelvis surgery'/exp OR 'pelvic ring':ti,ab) AND ('bone injury' / exp OR injur*:ti,ab } \\
\text { OR fractur*:ti,ab) AND ('three dimensional printing'/exp OR 'three-dimensional imaging'/exp OR } \\
\text { 3d:ti,ab OR 'three dimension"':ti,ab OR '3 dimension*':ti,ab OR navigation:ti,ab) AND [embase]/lim } \\
\text { AND (2010-2020)/py }\end{array}$ \\
\hline
\end{tabular}




\subsection{Inclusion and Exclusion Criteria}

Eligible studies for inclusion reported either on (1) the use of 3D techniques in the virtual planning of operative treatment of pelvic ring injuries; (2) 3D printed templates with fracture visualization; (3) 3D printed templates with pre-operative plate contouring; (4) 3D virtual planning of screw trajectories; (5) 3D custom-made implants with guides; and (6) 3D navigation for screw placement. Patients should be 18 years or older and patients with fragility fractures of the pelvis (FFP) were included as well. Outcomes directly related to the operative treatment should be reported. These included operation time, blood loss, screw malposition rate and fluoroscopy time or fluoroscopy frequency, fracture reduction and functional outcome. These outcome measures represent the efficiency and accuracy of the surgical procedure itself. We hypothesized that $3 \mathrm{D}$ assisted surgery could have an effect on these measures, which is the rationale to choose these outcome measures. Moreover, these are widely used for assessing pelvic ring surgery related to patient outcomes [9-11]. Except for case studies with $\mathrm{N}<10$ and conference abstracts, all study designs were accepted for inclusion. Concerning language, studies written in English, German, Spanish, French and Dutch were included. Biomechanical and animal studies were excluded, as well as studies about classification of injuries by means of 3D techniques. Moreover, studies that included outcomes after both pelvic ring injuries and acetabular fractures and that did not differentiate between these injuries in terms of outcomes were excluded.

\subsection{Study Selection}

All articles were imported into Rayyan QCRI, a web-based sorting tool for systematic literature reviews [12]. The study selection was performed in two screening phases: (1) title and abstract screening, and (2) full text screening. Both selection phases were independently performed by the same researchers. Disagreement was resolved by discussion. The initial searches (conducted from 1 January 2010 to 22 May 2020) generated 819 articles and after removal of duplicates, 709 potential eligible studies were screened. Following title and abstract assessment, 34 articles were reviewed in full text. A total of 18 articles were included in the review of which most were case-control studies $(\mathrm{N}=9)$, followed by cross-sectional cohort studies $(\mathrm{N}=8)$ and one prospective cohort study. No randomized controlled trials (RCTs) were found in this search. Figure 1 demonstrates a flowchart of the inclusion procedure.

\subsection{Data Extraction}

The data extraction was independently conducted (HB, FIJ) using a precompiled extraction file (Microsoft Excel version 14.0; Microsoft Inc., Redmond, WA, USA). Study characteristics, fracture classification, 3D technologies and outcome measures were extracted from all the included studies by the senior author.

\subsection{Assessment of Methodological Quality}

Methodological quality and risk of bias of the included studies was independently assessed according to the guidelines of the McMaster University Occupational Therapy Evidence-Based Practice Research Group [13]. The Modified McMaster Critical Review form consists of nine categories: citation, study purpose, literature, design, sample, outcomes, intervention, results, and conclusions and implications. This review form is appropriate to assess RCTs, cohort studies, single-case designs, before- and after-designs, case control studies, cross-sectional studies and case studies. The guidelines established by Law et al. [13] were utilized for the quality assessment. Every item was answered with 'yes; 1 point', 'no; 0 points', 'not addressed; 0 points' or 'not applicable (N/A); no points given'. Any continued disagreements were solved during a consensus meeting (HB and FIJ). The total score reflects the methodological quality with a maximum score of 17 for RCTs and 13 for other designs. The definitive score is calculated in a percentage and may vary from $0-100 \%$, with a higher score indicating a higher methodological quality. Scores below $<60 \%$ were considered as poor quality, scores between $60-74 \%$ were considered 
as moderate quality, scores between $75-89 \%$ indicated good-quality and scores between 90-100\% indicated excellent-quality studies.

\section{PRISMA 2009 Flow Diagram}
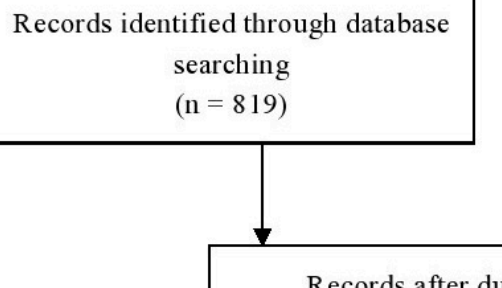

Records after duplicates removed

$(\mathrm{n}=709)$
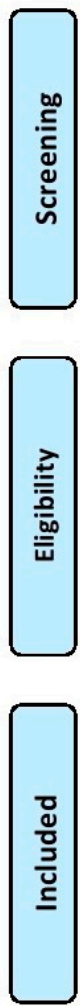

Additional records identified through other sources $(\mathrm{n}=0)$

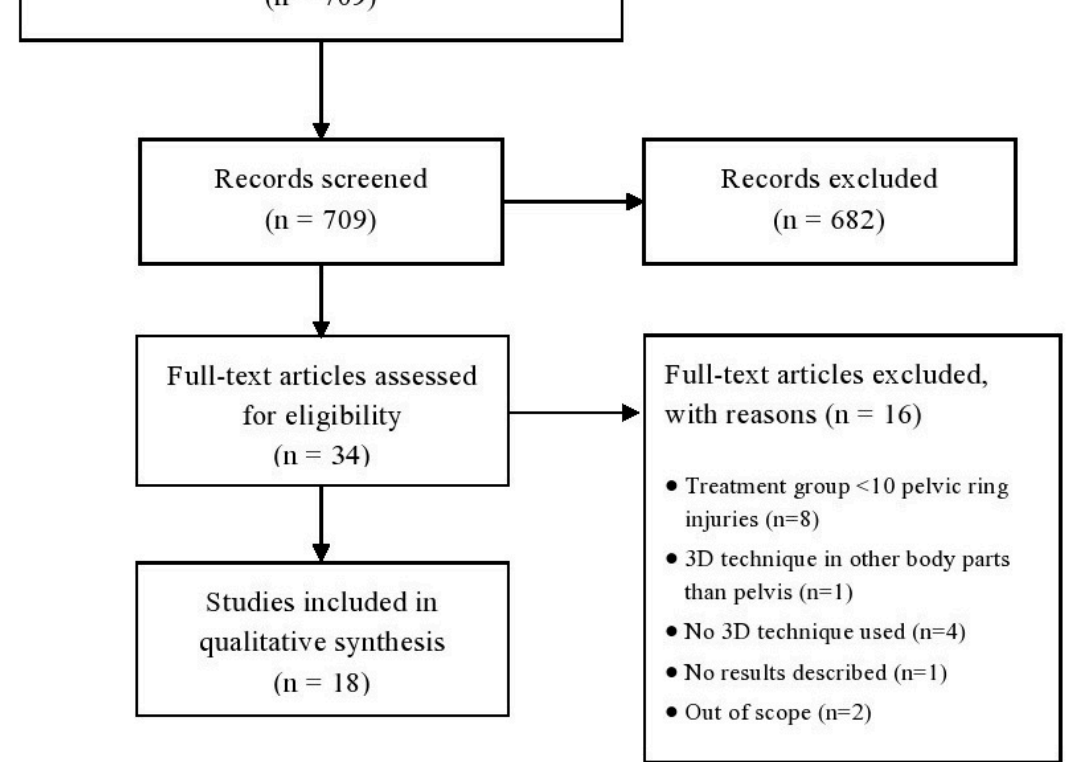

Figure 1. Flow diagram according to the PRISMA method.

The results of the quality assessment of the included articles are presented in Table 2. A maximum score of 13 could be obtained as four items concerning RCTs were left out. Total scores in percentages ranged between $46 \%$ and $92 \%$ with a mean score of $63 \%$ (SD 16). Only one study was considered as excellent quality, five were good-quality, four moderate quality and eight poor quality studies. 
Table 2. Scores of the quality assessment list ranged from best to worst score.

\begin{tabular}{|c|c|c|c|c|c|c|c|c|c|c|c|c|c|c|c|}
\hline- & 1 & 2 & 3 & 4 & 5 & 6 & 7 & 8 & 9 & 10 & 11 & 12 & 13 & Total & $\%$ \\
\hline & Citation & $\begin{array}{l}\text { Study } \\
\text { Purpose }\end{array}$ & $\begin{array}{c}\text { Literature } \\
\text { Review }\end{array}$ & & ple & $\mathrm{Ou}$ & mes & Intervention & & $\operatorname{Re}$ & alts & & $\begin{array}{l}\text { Conclusion } \\
\text { and Clinical } \\
\text { Implications }\end{array}$ & & \\
\hline $\begin{array}{l}\text { Berger-Groch } \\
\text { et al. [14] }\end{array}$ & + & + & + & + & - & + & + & + & + & + & + & + & + & $12 / 13$ & 92 \\
\hline Takao et al. [15] & + & + & + & + & - & + & + & + & + & + & + & - & + & $11 / 13$ & 85 \\
\hline Yang et al. [16] & + & + & + & + & - & + & + & + & + & + & + & - & + & $11 / 13$ & 85 \\
\hline Hung et al. [3] & + & + & + & + & - & + & - & + & + & + & + & - & + & $10 / 13$ & 76 \\
\hline Li. et al. [17] & + & + & + & + & - & + & - & + & + & + & + & - & + & $10 / 13$ & 76 \\
\hline Teo et al. [18] & + & + & + & + & - & + & + & + & + & - & + & - & + & $10 / 13$ & 76 \\
\hline Cai et al. [4] & + & + & + & + & - & - & - & + & + & + & + & - & + & $9 / 13$ & 69 \\
\hline Li et al. [19] & + & + & + & + & - & - & - & + & + & + & + & - & + & $9 / 13$ & 69 \\
\hline Balling [6] & + & + & + & + & - & + & + & + & - & - & + & - & - & $8 / 13$ & 62 \\
\hline Takeba et al. [20] & + & + & + & + & - & + & + & + & - & - & + & - & - & $8 / 13$ & 62 \\
\hline Pieske et al. [21] & + & + & + & + & - & - & - & + & + & - & + & - & - & $7 / 13$ & 54 \\
\hline Beck et al. [22] & + & + & + & + & - & - & - & + & - & - & + & - & - & $6 / 13$ & 46 \\
\hline Chen et al. [23] & + & + & + & + & - & - & - & + & - & - & + & - & - & $6 / 13$ & 46 \\
\hline Gao et al. [24] & + & + & + & + & - & - & - & + & - & - & + & - & - & $6 / 13$ & 46 \\
\hline Ghisla et al. [25] & + & + & + & + & - & - & - & + & - & - & + & - & - & $6 / 13$ & 46 \\
\hline Kim et al. [26] & + & + & + & + & - & - & - & + & - & - & + & - & - & $6 / 13$ & 46 \\
\hline Nie et al. [27] & + & - & + & + & - & - & - & + & + & - & + & - & - & $6 / 13$ & 46 \\
\hline $\begin{array}{l}\text { Privalov et al. } \\
\text { [28] }\end{array}$ & + & + & + & - & - & - & - & + & + & - & + & - & - & $6 / 13$ & 46 \\
\hline
\end{tabular}

Every plus (+) sign means that the question was answered with 'yes'. Every minus (-) sign means that a question was answered with 'no' or 'not addressed'. The final two columns represent the total scores and percentages of maximal attainable scores $(\%)$.

\subsection{Outcomes}

Outcomes relevant to the operation were recorded. These parameters included operation time, blood loss, screw malposition (varying from contacting cortical bone to actual perforation of the cortical bone), fluoroscopy dose, amount and frequency, fracture reduction according to the guidelines established by Tornetta and Matta [29] and patient- or physician-reported functional outcome.

\subsection{Patient and Injury Characteristics}

Overall, data of a total of 988 patients were reported in the studies (Table 3). Most studies $(\mathrm{N}=12)$ focused on unstable pelvic ring injuries (Type $\mathrm{B}$ and Type $\mathrm{C}$ according to the AO classification system) [30]. Of all included patients, 694 received 3D-assisted pelvic ring injury surgery and 294 had conventional surgery. The study characteristics are shown in Table 3.

\subsection{Strategy for Data Synthesis and Statistical Analysis}

Data synthesis involved the comparison, combination, and summary of findings. Data are presented as part of a narrative synthesis, involving text and tables. Continuous variables are presented as means with standard deviation (SD) (parametric data) or as median with interquartile range (IQR) in case of non-parametric data. Dichotomous variables are given as frequency and percentages. Due to the retrospective nature of the included studies and the heterogeneity of their design, results could not be pooled for statistical analysis. Instead, weighted means of the various outcome variables of the studies were calculated for comparison. Besides, a best-evidence synthesis was performed, taking into account the methodological quality and outcome of the original studies (Table 4) [31]. Excellent and good quality studies were labeled as high-quality studies whereas moderate and low-quality studies were labeled as low-quality. 
Table 3. Study characteristics.

\begin{tabular}{|c|c|c|c|c|c|c|c|}
\hline No. & Study & Year & $\mathbf{N}$ & Method * & $\begin{array}{l}\text { Study } \\
\text { Period }\end{array}$ & Injury Type & Intervention \\
\hline 1 & Balling [6] & 2019 & 52 & CSS & 2011-2016 & Sacral FFPs ${ }^{\circledR}$ & $\begin{array}{l}\text { 3D image guided sacral screw fixation via } \\
\text { single-sided minimally invasive } \\
\text { transgluteal approach }\end{array}$ \\
\hline 2 & Beck et al. [22] & 2010 & 26 & CCS & 2008-2009 & AO/Tile B, C & $\begin{array}{l}\text { S: intra-operative 3D fluoroscopy of } \\
\text { iliosacral screws and lumbopelvic } \\
\text { implants }(\mathrm{N}=14) \\
\text { C: iliosacral screws and lumbopelvic } \\
\text { implants without intra-operative } \\
\text { 3D }(\mathrm{N}=12)\end{array}$ \\
\hline 3 & $\begin{array}{l}\text { Berger-Groch } \\
\text { et al. [14] }\end{array}$ & 2018 & 136 & CCS & 2004-2014 & AO/Tile B, C & $\begin{array}{l}\text { S: 3D navigated iliosacral screw placement } \\
(\mathrm{N}=100) \\
\text { C: conventional iliosacral screw placement } \\
(\mathrm{N}=36)\end{array}$ \\
\hline 4 & Cai et al. [4] & 2018 & 137 & CCS & 2014-2016 & AO/Tile B, C & $\begin{array}{l}\text { S: 3D printing-based minimally invasive } \\
\text { cannulated screw treatment }(\mathrm{N}=65) \\
\text { C: conventional surgery without 3D } \\
\text { printing }(\mathrm{N}=72)\end{array}$ \\
\hline 5 & Chen et al. [23] & 2019 & 28 & PCS & 2016-2018 & AO/Tile B, C & $\begin{array}{l}\text { Minimally invasive screw fixation using } \\
\text { the "Blunt End" Kirschner wire technique } \\
\text { assisted by 3D printed external template }\end{array}$ \\
\hline 6 & Gao et al. [24] & 2011 & 22 & CSS & 2006-2008 & AO/Tile B, C & $\begin{array}{l}\text { Minimally invasive fluoro-navigation } \\
\text { screw fixation }\end{array}$ \\
\hline 7 & Ghisla et al. [25] & 2018 & 21 & CSS & 2008-2017 & $\begin{array}{l}\text { Posterior } \\
\text { pelvic ring }\end{array}$ & $\begin{array}{l}\text { Intra-operative 3D-CT guided navigation } \\
\text { for iliosacral screws }\end{array}$ \\
\hline 8 & Hung et al. [3] & 2018 & 30 & CCS & 2012-2017 & $\begin{array}{l}\mathrm{AO} / \text { Tile A, } \\
\mathrm{B}, \mathrm{C}\end{array}$ & $\begin{array}{l}\text { S: ORIF with pre-operative virtual } \\
\text { simulation and 3D- printing-assisted } \\
\text { contoured plate }(\mathrm{N}=16) \\
\text { C: ORIF with conventional plate fixation } \\
(\mathrm{N}=14)\end{array}$ \\
\hline 9 & Kim et al. [26] & 2013 & 29 & CSS & 2010 & AO/Tile A, B & $\begin{array}{l}\text { Percutaneous iliosacral screwing using } \\
\text { 3D-fluoroscopy }\end{array}$ \\
\hline 10 & Li et al. [19] & 2015 & 157 & CCS & 2009-2014 & AO/Tile C & $\begin{array}{c}\text { S: computer-aided angiography and rapid } \\
\text { prototyping technology }(\mathrm{N}=81) \\
\text { C: conventional imaging }(\mathrm{N}=76)\end{array}$ \\
\hline 11 & Li. et al. [17] & 2015 & 81 & CCS & 2005-2011 & AO/Tile B, C & $\begin{array}{c}\text { S: 3D C-arm fluoroscopy navigation } \\
(\mathrm{N}=43) \\
\text { C: C-arm fluoroscopy }(\mathrm{N}=38)\end{array}$ \\
\hline 12 & Nie et al. [27] & 2018 & 30 & CSS & 2015-2017 & AO/Tile B, C & $\begin{array}{l}\text { 3D printing assisted by minimally } \\
\text { invasive surgery for pubic rami fractures }\end{array}$ \\
\hline 13 & Pieske et al. [21] & 2015 & 71 & CSS & Unknown & AO/Tile B, C & $\begin{array}{l}\text { CT-guided sacroiliac percutaneous screw } \\
\text { placement }\end{array}$ \\
\hline 14 & $\begin{array}{l}\text { Privalov et al. } \\
{[28]}\end{array}$ & 2020 & 53 & CCS & 2017-2018 & $\begin{array}{l}\text { Posterior } \\
\text { pelvic ring }\end{array}$ & $\begin{array}{c}\text { S: intra-operative } \mathrm{CT} \text { in navigated } \\
\text { sacroiliac instrumentation }(\mathrm{N}=25) \\
\mathrm{C}^{1} \text { : navigated surgery with } \\
\text { intra-operative 3D-C-Arm }(\mathrm{N}=15) \\
\mathrm{C}^{2} \text { : conventional surgery with } \\
\text { intra-operative control by 3D-C-Arm } \\
(\mathrm{N}=9) \\
\mathrm{C}^{3} \text { : conventional surgery with } \\
\text { intra-operative control by 2D fluoroscopy } \\
(\mathrm{N}=4)\end{array}$ \\
\hline 15 & Takao et al. [15] & 2019 & 27 & CSS & 2011-2016 & AO/Tile B, C & $\begin{array}{l}\text { 3D fluoroscopic navigation of iliosacral } \\
\text { screw insertion }\end{array}$ \\
\hline 16 & Takeba et al. [20] & 2018 & 10 & CSS & 2013-2017 & AO/Tile B, C & $\begin{array}{l}\text { O-arm and stealth station navigation for } \\
\text { screw fixation }\end{array}$ \\
\hline 17 & Teo et al. [18] & 2018 & 36 & CCS & 2011-2016 & AO/Tile B, C & $\begin{array}{l}\text { S: sacroiliac screw placement with } \\
\text { intra-operative navigation C: sacroiliac } \\
\text { screw placement without } \\
\text { intra-operative navigation }\end{array}$ \\
\hline 18 & Yang et al. [16] & 2018 & 40 & CCS & 2016-2017 & AO/Tile B, C & $\begin{array}{l}\text { S: 3D printed external template to guide } \\
\text { iliosacral screw insertion }(\mathrm{N}=22) \\
\text { C: conventional without external template } \\
\qquad(\mathrm{N}=18)\end{array}$ \\
\hline
\end{tabular}

${ }^{*}$ CSS, cross-sectional study; PCS, prospective cohort study; CCS, case-control study; S, study group; C, control group ${ }^{\circledR}$ FFP, fragility fracture of the pelvis. 
Table 4. Best-evidence synthesis.

\begin{tabular}{cc}
\hline & Best-Evidence Synthesis \\
\hline $\begin{array}{c}\text { Strong evidence } \\
\text { Moderate evidence }\end{array}$ & $\begin{array}{c}\text { Consistent findings among multiple high-quality studies } \\
\text { Consistent findings in multiple low-quality studies and/or one } \\
\text { high-quality study } \\
\text { Cimited evidence } \\
\text { Conflicting evidence } \\
\text { Consistent findings in at least one low-quality study } \\
\text { Inconsistent findings among multiple studies (high- and/or } \\
\text { low-quality studies) }\end{array}$ \\
$\begin{array}{c}\text { Findings of eligible studies do not meet the criteria for one of the levels } \\
\text { of evidence stated above, or there are no eligible studies available }\end{array}$ \\
\hline
\end{tabular}

\section{Results}

\subsection{Intra-Operative Results}

Our first question asks about the difference in intra-operative results in terms of operation time, blood loss and fluoroscopy time between 3D-assisted and conventional (2D) surgery. All identified 3D-assisted surgery techniques are shown in Figure 2 and described in Supplemental Digital Content 1.

3D visualization and Sacro-Iliac screw planning:
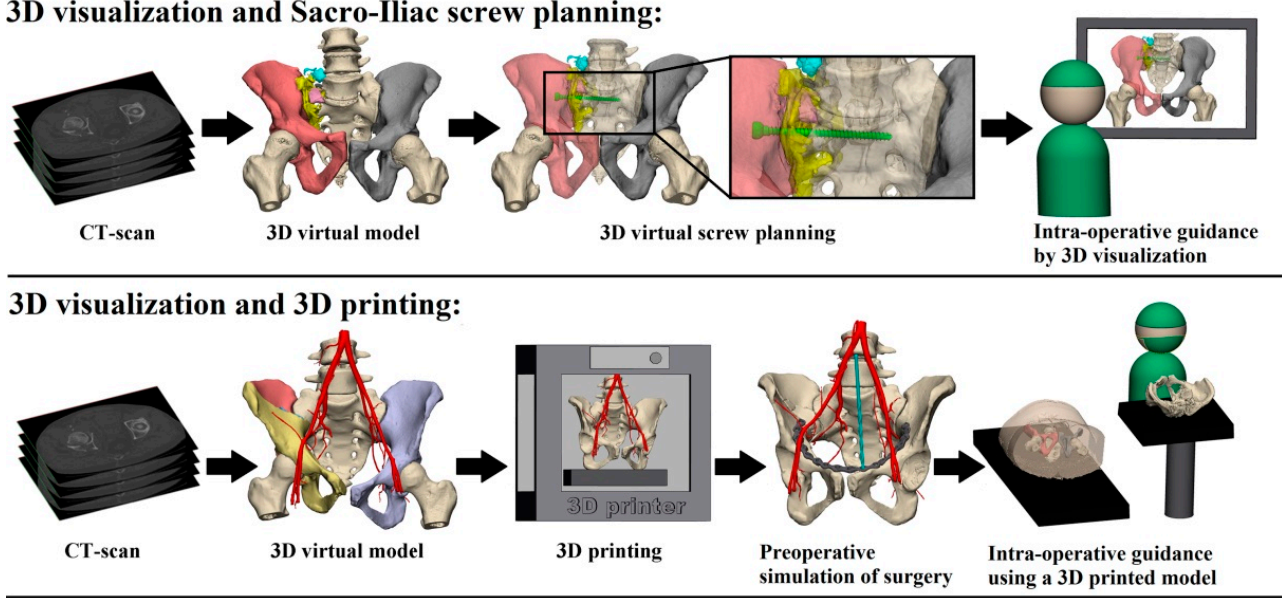

3D printing and pre-contouring of the implant:

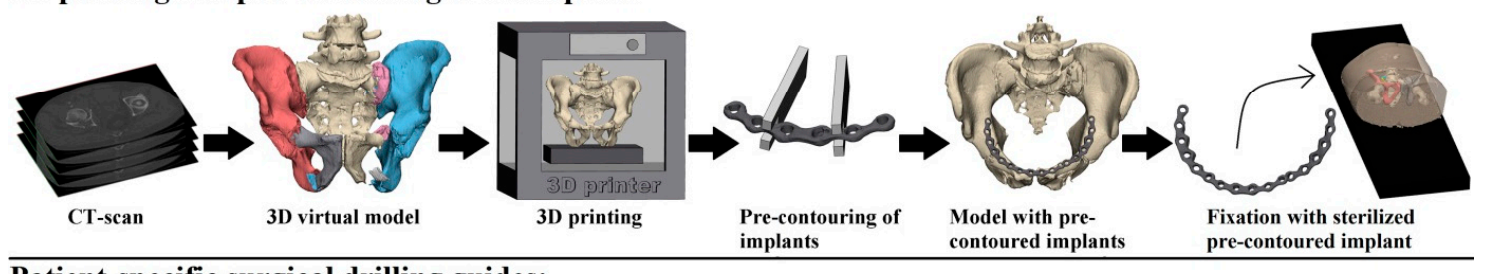

Patient-specific surgical drilling guides:
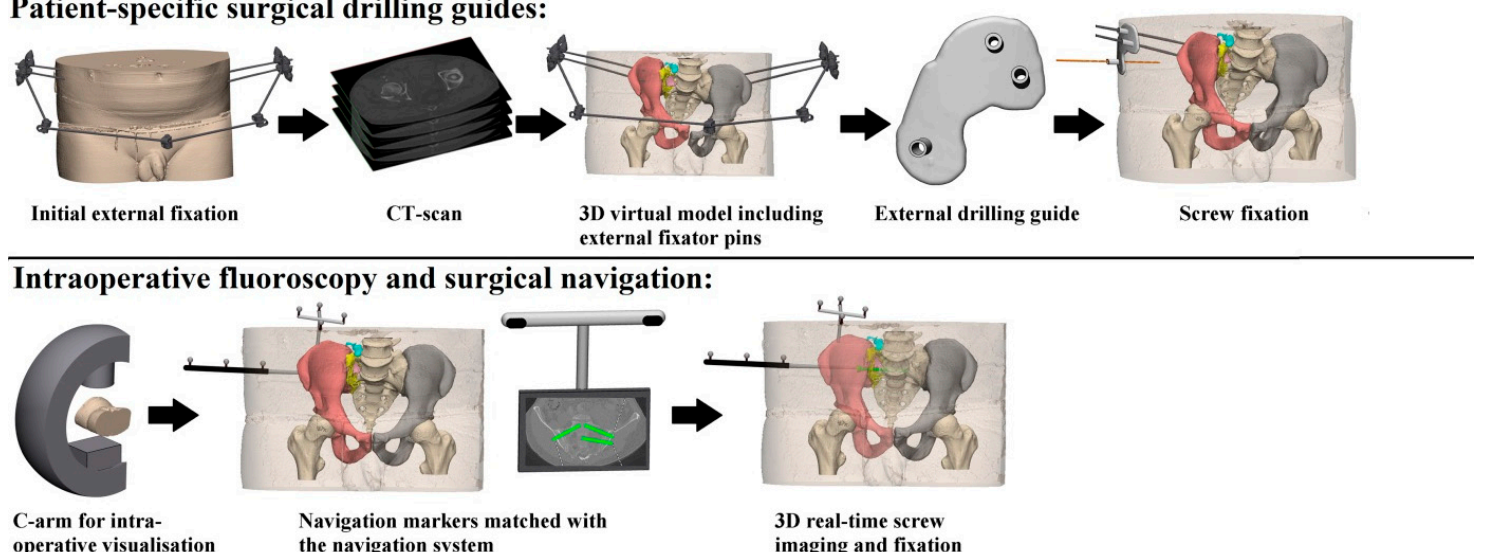

Figure 2. Presentation of the five identified 3D-assisted surgery techniques. 


\subsubsection{Operation Time per Screw and Overall Operation Time}

Two out of three case-control studies reported that 3D-assisted surgery led to a significant decrease in operation time per screw using 3D printed drilling guides [16] and intra-operative 3D imaging [17] (Table 5). Berger-Groch et al. [14] found no difference using intra-operative 3D imaging. Weighted mean operation time per screw was $15 \mathrm{~min}$ (range 14-18) for 3D-assisted and 26 min (range 19-40) for the conventional group.

Table 5. Study outcomes.

\begin{tabular}{|c|c|c|c|c|c|c|c|}
\hline \multirow[t]{2}{*}{ Measure } & \multirow[t]{2}{*}{ Study } & \multirow[t]{2}{*}{ 3D Technology } & \multicolumn{2}{|c|}{ Groups (N) } & \multicolumn{3}{|c|}{ Outcomes } \\
\hline & & & $3 \mathrm{D}$ & Conventional & $3 \mathrm{D}$ & Conventional & $p$-Value \\
\hline \multicolumn{8}{|c|}{ Intra-Operative Results } \\
\hline \multirow{8}{*}{$\begin{array}{l}\text { Operation time per } \\
\text { screw (min) } \\
\text { Mean } \pm \text { std or } \\
\text { Mean } \pm(\text { range })\end{array}$} & $\begin{array}{c}\text { Berger-Groch et al. } \\
{[14]}\end{array}$ & $\begin{array}{l}\text { 3D navigated iliosacral } \\
\text { screw placement }\end{array}$ & 100 & 36 & $48 \pm 25$ & $50 \pm 29$ & 0.74 \\
\hline & Chen et al. [23] & $\begin{array}{l}\text { Minimally invasive screw } \\
\text { fixation using the "Blunt } \\
\text { End" kirschner wire } \\
\text { technique assisted by 3D } \\
\text { printed external template }\end{array}$ & 28 & - & $21 \pm 3$ & - & - \\
\hline & Gao et al. [24] & $\begin{array}{l}\text { Minimally invasive } \\
\text { fluoro-navigation } \\
\text { screw fixation }\end{array}$ & 22 & - & $24(16-45)$ & - & - \\
\hline & Kim et al. [26] & $\begin{array}{l}\text { Percutaneous iliosacral } \\
\text { screwing using } \\
\text { 3D-fluoroscopy }\end{array}$ & 29 & - & $36(18-83)$ & - & - \\
\hline & Li. et al. [17] & $\begin{array}{l}\text { Percutaneous screw fixation } \\
\text { using three-dimensional } \\
\text { (ISO-C3D) navigation }\end{array}$ & 43 & 38 & $14 \pm 1$ & $19 \pm 1$ & $<0.001$ \\
\hline & Pieske et al. [21] & $\begin{array}{l}\text { CT-guided sacroiliac } \\
\text { percutaneous screw } \\
\text { placement }\end{array}$ & 71 & - & $63 \pm 39$ & - & - \\
\hline & Takeba et al. [20] & $\begin{array}{l}\text { O-arm and stealthstation } \\
\text { navigation for } \\
\text { screw fixation }\end{array}$ & 10 & - & $39(25-68)$ & - & - \\
\hline & Yang et al. [16] & $\begin{array}{l}\text { 3D printed external } \\
\text { template to guide iliosacral } \\
\text { screw insertion }\end{array}$ & 22 & 18 & $18 \pm 5$ & $40 \pm 11$ & $<0.001$ \\
\hline \multirow{5}{*}{$\begin{array}{l}\text { Operation time } \\
\text { overall (min) } \\
\text { Mean } \pm \text { std }\end{array}$} & Cai et al. [4] & $\begin{array}{l}\text { 3D printing-based } \\
\text { minimally invasive } \\
\text { cannulated screw treatment }\end{array}$ & 65 & 72 & $59 \pm 13$ & $72 \pm 13$ & $<0.001$ \\
\hline & Chen et al. [23] & $\begin{array}{l}\text { Minimally invasive screw } \\
\text { fixation using the "Blunt } \\
\text { End" kirschner wire } \\
\text { technique assisted by 3D } \\
\text { printed external template }\end{array}$ & 28 & - & $85(60-150)$ & - & - \\
\hline & Hung et al. [3] & $\begin{array}{l}\text { Pre-operative virtual } \\
\text { simulation and 3D } \\
\text { printing-assisted } \\
\text { contoured plate }\end{array}$ & 16 & 14 & $206 \pm 70$ & $276 \pm 90$ & 0.023 \\
\hline & Li et al. [19] & $\begin{array}{l}\text { Computer-aided } \\
\text { angiography and rapid } \\
\text { prototyping technology }\end{array}$ & 81 & 76 & $105 \pm 19$ & $122 \pm 23$ & 0.035 \\
\hline & Privalov et al. [28] & $\begin{array}{l}\text { Intra-operative CT in } \\
\text { navigated sacroiliac } \\
\text { instrumentation }\end{array}$ & 25 & 28 & $189 \pm 89$ & $\begin{array}{l}C^{1}: 153 \pm 68 \\
C^{2}: 201 \pm 100 \\
C^{3}: 127 \pm 70\end{array}$ & $\begin{array}{l}0.31 \\
0.70 \\
0.14\end{array}$ \\
\hline
\end{tabular}


Table 5. Cont.

\begin{tabular}{|c|c|c|c|c|c|c|c|}
\hline \multirow[t]{2}{*}{ Measure } & \multirow[t]{2}{*}{ Study } & \multirow[t]{2}{*}{ 3D Technology } & \multicolumn{2}{|c|}{ Groups (N) } & \multicolumn{3}{|c|}{ Outcomes } \\
\hline & & & $3 \mathrm{D}$ & Conventional & $3 \mathrm{D}$ & Conventional & $p$-Value \\
\hline \multirow{3}{*}{$\begin{array}{c}\text { Blood loss } \\
(\mathrm{mL}) \text { Mean } \pm \text { std or } \\
\text { Mean (range) }\end{array}$} & Hung et al. [3] & $\begin{array}{l}\text { pre-operative virtual } \\
\text { simulation and 3D } \\
\text { printing-assisted } \\
\text { contoured plate }\end{array}$ & 16 & 14 & $275 \pm 197$ & $549 \pm 404$ & 0.023 \\
\hline & Nie et al. [27] & $\begin{array}{l}\text { 3D printing assisted by } \\
\text { minimally invasive surgery }\end{array}$ & 30 & - & $31 \pm 11$ & - & - \\
\hline & Takeba et al. [20] & $\begin{array}{l}\text { O-arm and stealthstation } \\
\text { navigation for } \\
\text { screw fixation }\end{array}$ & 10 & - & $12(0-120)$ & - & - \\
\hline \multirow{5}{*}{$\begin{array}{l}\text { Fluoroscopy Dose } \\
\text { mean } \pm \text { SD or } \\
\text { mean (range) } \\
\text { presented in the } \\
\text { given unit }\end{array}$} & Balling [6] & $\begin{array}{l}\text { 3D image guided sacral } \\
\text { screw fixation via } \\
\text { single-sided minimally } \\
\text { invasive transgluteal } \\
\text { approach }\end{array}$ & 52 & - & $\begin{array}{c}788 \pm \\
632 \mathrm{mGy} / \mathrm{cm}\end{array}$ & - & - \\
\hline & Beck et al. [22] & $\begin{array}{c}\text { Intra-operative 3D } \\
\text { fluoroscopy of iliosacral } \\
\text { screws and } \\
\text { lumbopelvic implants }\end{array}$ & 14 & 12 & $\begin{array}{l}181 \mathrm{cGy} / \mathrm{cm}^{2} \\
(90-424)\end{array}$ & $\begin{array}{c}1376 \mathrm{cGy} / \mathrm{cm}^{2} \\
(485-2)\end{array}$ & NA \\
\hline & Ghisla et al. [25] & $\begin{array}{l}\text { Intra-operative 3D-CT } \\
\text { guided navigation for } \\
\text { sacro-iliac screws }\end{array}$ & 21 & - & $\begin{array}{l}1918 \\
\mathrm{mGy} / \mathrm{cm}\end{array}$ & - & - \\
\hline & Pieske et al. [21] & $\begin{array}{l}\text { CT-guided sacroiliac } \\
\text { percutaneous screw } \\
\text { placement }\end{array}$ & 71 & - & $\begin{array}{c}\text { Male: } 6 \pm 3 \\
\text { msV, range: } \\
2-17 \text {; Female: } \\
9 \pm 3 \mathrm{msV} \\
\text { range: } 1-28\end{array}$ & - & - \\
\hline & Yang et al. [16] & $\begin{array}{l}\text { 3D printed external } \\
\text { template to guide iliosacral } \\
\text { screw insertion }\end{array}$ & 22 & 18 & $\begin{array}{l}743 \pm 231 \\
\mathrm{cGy} / \mathrm{cm}^{2}\end{array}$ & $\begin{array}{c}1904 \pm 845 \\
\mathrm{cGy} / \mathrm{cm}^{2}\end{array}$ & $<0.001$ \\
\hline \multirow{6}{*}{$\begin{array}{l}\text { Fluoroscopy time } \\
\text { (sec) mean } \pm \text { SD or } \\
\text { mean (range) }\end{array}$} & Beck et al. [22] & $\begin{array}{c}\text { Intra-operative 3D } \\
\text { fluoroscopy of iliosacral } \\
\text { screws and } \\
\text { lumbopelvic implants }\end{array}$ & 14 & 12 & $64(60-71)$ & $181(54-340)$ & NA \\
\hline & $\begin{array}{l}\text { Berger-Groch et al. } \\
{[14]}\end{array}$ & $\begin{array}{l}\text { 3D navigated iliosacral } \\
\text { screw placement }\end{array}$ & 100 & 36 & $99 \pm 812$ & $164 \pm 166$ & 0.02 \\
\hline & Gao et al. [24] & $\begin{array}{l}\text { Minimally invasive } \\
\text { fluoro-navigation } \\
\text { screw fixation }\end{array}$ & 22 & - & $22(10-46)$ & - & - \\
\hline & Kim et al. [26] & $\begin{array}{l}\text { Percutaneous iliosacral } \\
\text { screwing using } \\
\text { 3D-fluoroscopy }\end{array}$ & 29 & - & $84(22-160)$ & & \\
\hline & Li. et al. [17] & $\begin{array}{l}\text { Percutaneous screw fixation } \\
\text { using three-dimensional } \\
\text { (ISO-C3D) navigation }\end{array}$ & 43 & 38 & $34 \pm 2$ & $58 \pm 5$ & $<0.001$ \\
\hline & Privalov et al. [28] & $\begin{array}{l}\text { Intra-operative CT in } \\
\text { navigated sacroiliac } \\
\text { instrumentation }\end{array}$ & 25 & 28 & $82 \pm 97$ & $\begin{array}{l}C^{1}: 299 \pm 374 \\
C^{2}: 243 \pm 92 \\
C^{3}: 248 \pm 191\end{array}$ & $\begin{array}{l}0.03 \\
0.00 \\
0.02\end{array}$ \\
\hline \multirow{2}{*}{$\begin{array}{c}\text { Fluoroscopy } \\
\text { frequency number } \\
\text { of times in mean } \pm \\
\text { SD or mean (range) }\end{array}$} & Cai et al. [4] & $\begin{array}{l}\text { 3D printing-based } \\
\text { minimally invasive } \\
\text { cannulated screw treatment }\end{array}$ & 65 & 72 & $29 \pm 4$ & $37 \pm 3$ & $<0.001$ \\
\hline & Chen et al. [23] & $\begin{array}{l}\text { Minimally invasive screw } \\
\text { fixation using the "Blunt } \\
\text { End" kirschner wire } \\
\text { technique assisted by 3D } \\
\text { printed external template }\end{array}$ & 28 & - & $35(28-60)$ & - & - \\
\hline
\end{tabular}


Table 5. Cont.

\begin{tabular}{|c|c|c|c|c|c|c|c|}
\hline \multirow[t]{2}{*}{ Measure } & \multirow[t]{2}{*}{ Study } & \multirow[t]{2}{*}{ 3D Technology } & \multicolumn{2}{|c|}{ Groups (N) } & \multicolumn{3}{|c|}{ Outcomes } \\
\hline & & & $3 \mathrm{D}$ & Conventional & $3 D$ & Conventional & $p$-Value \\
\hline \multicolumn{8}{|c|}{ Post-Operative Results } \\
\hline \multirow{11}{*}{$\begin{array}{l}\text { Screw malposition } \\
\text { rate }(\%)\end{array}$} & Beck et al. [22] & $\begin{array}{c}\text { Intra-operative 3D } \\
\text { fluoroscopy of iliosacral } \\
\text { screws and } \\
\text { lumbopelvic implants }\end{array}$ & 14 & 12 & 7 & 6 & NA \\
\hline & Gao et al. [24] & $\begin{array}{l}\text { Minimally invasive } \\
\text { fluoro-navigation } \\
\text { screw fixation }\end{array}$ & 22 & - & 2 & - & - \\
\hline & Ghisla et al. [25] & $\begin{array}{l}\text { Intra-operative 3D-CT } \\
\text { guided navigation for } \\
\text { sacro-iliac screws }\end{array}$ & 21 & - & 3 & - & - \\
\hline & Kim et al. [26] & $\begin{array}{l}\text { Percutaneous iliosacral } \\
\text { screwing using } \\
\text { 3D-fluoroscopy }\end{array}$ & 29 & - & 23 & - & - \\
\hline & Li. et al. [17] & $\begin{array}{l}\text { Percutaneous screw fixation } \\
\text { using three-dimensional } \\
\text { (ISO-C3D) navigation }\end{array}$ & 43 & 38 & 5 & 24 & 0.015 \\
\hline & Pieske et al. [21] & $\begin{array}{c}\text { CT-guided sacroiliac } \\
\text { percutaneous screw } \\
\text { placement }\end{array}$ & 71 & - & 1 & - & - \\
\hline & Takao et al. [15] & $\begin{array}{l}\text { 3D fluoroscopic navigation } \\
\text { of iliosacra screw insertion }\end{array}$ & 27 & - & 7 & - & - \\
\hline & Takeba et al. [20] & $\begin{array}{l}\text { O-arm and stealthstation } \\
\text { navigation for } \\
\text { screw fixation }\end{array}$ & 10 & - & 0 & - & - \\
\hline & Teo et al. [18] & $\begin{array}{l}\text { Sacroiliac screw placement } \\
\text { with and without } \\
\text { intra-operative navigation }\end{array}$ & 17 & 19 & 12 & 5 & 0.48 \\
\hline & Yang et al. [16] & $\begin{array}{l}\text { 3D printed external } \\
\text { template to guide iliosacral } \\
\text { screw insertion }\end{array}$ & 22 & 18 & 3 & 14 & $<0.001$ \\
\hline & $\begin{array}{c}\text { Berger-Groch et al. } \\
{[14]}\end{array}$ & $\begin{array}{l}\text { 3D navigated iliosacral } \\
\text { screw placement }\end{array}$ & 100 & 36 & 14 & 21 & 0.09 \\
\hline \multirow{4}{*}{$\begin{array}{c}\text { Reduction } \\
\text { according to Matta } \\
\text { (excellent + good in } \\
\%)\end{array}$} & Cai et al. [4] & $\begin{array}{l}\text { 3D printing-based } \\
\text { minimally invasive } \\
\text { cannulated screw treatment }\end{array}$ & 65 & 72 & 79 & 81 & 0.762 \\
\hline & Chen et al. [23] & $\begin{array}{l}\text { Minimally invasive screw } \\
\text { fixation using the "Blunt } \\
\text { End" kirschner wire } \\
\text { technique assisted by 3D } \\
\text { printed external template }\end{array}$ & 28 & - & 89 & - & - \\
\hline & Nie et al. [27] & $\begin{array}{l}\text { 3D printing assisted by } \\
\text { minimally invasive surgery } \\
\text { for pubic rami fractures }\end{array}$ & 30 & - & 100 & - & - \\
\hline & Yang et al. [16] & $\begin{array}{l}\text { 3D printed external } \\
\text { template to guide iliosacral } \\
\text { screw insertion }\end{array}$ & 22 & 18 & 86 & 89 & 1.000 \\
\hline \multirow{4}{*}{$\begin{array}{l}\text { Functional } \\
\text { outcome (Majeed } \\
\text { excellent }+ \text { good } \\
\text { rate in } \% \text { ) }\end{array}$} & Cai et al. [4] & $\begin{array}{l}\text { 3D printing-based } \\
\text { minimally invasive } \\
\text { cannulated screw treatment }\end{array}$ & 65 & 72 & 82 & 81 & 0.884 \\
\hline & Chen et al. [23] & $\begin{array}{l}\text { Minimally invasive screw } \\
\text { fixation using the "Blunt } \\
\text { End" kirschner wire } \\
\text { technique assisted by 3D } \\
\text { printed external template }\end{array}$ & 28 & - & 82 & - & - \\
\hline & Li. et al. [17] & $\begin{array}{l}\text { Percutaneous screw fixation } \\
\text { using three-dimensional } \\
\text { (ISO-C3D) navigation }\end{array}$ & 43 & 38 & 92 & 89 & 0.637 \\
\hline & Nie et al. [27] & $\begin{array}{l}\text { 3D printing assisted by } \\
\text { minimally invasive surgery } \\
\text { for pubic rami fractures }\end{array}$ & 30 & - & 100 & - & - \\
\hline
\end{tabular}


Three case-control studies reported on a significant decrease in overall operation time using a 3D printed model [4,19] and a 3D printed model combined with pre-contouring of the osteosynthesis plate [3] compared to the conventional technique. The other case-control study found no difference using intra-operative 3D imaging for screw placement [28]. Weighted mean overall operation time was $97 \mathrm{~min}$ (range 59-206) for 3D-assisted and 113 min (range 72-276) for the conventional group.

\subsubsection{Blood Loss}

The only case-control study by Hung et al. [3] reported a significant decrease in blood loss (275 $\pm 197 \mathrm{~mL}$ versus $549 \pm 404 \mathrm{~mL} ; p=0.023)$ using pre-operative virtual simulation and $3 \mathrm{D}$ printing-assisted plate contouring. No weighted mean blood loss of all studies was calculated as both open and percutaneous surgery was applied.

\subsubsection{Fluoroscopy Dose, Time and Frequency}

One case-control study by Yang et al. [16] reported a significantly decreased fluoroscopy dose by using 3D printed drilling guides in comparison to conventional surgery. The other case-control study by Beck et al. [22] did not express the difference in fluoroscopy dose in a $p$-value. No weighted mean could be calculated because different units were used to report dose.

Three of the four case-control studies reported that intra-operative 3D-assisted surgery significantly reduced fluoroscopy time $[14,17,28]$. The remaining case-control study by Beck et al. [22] did not express the difference in fluoroscopy time with a $p$-value. Weighted mean fluoroscopy time was 74 s (range 22-29) for the 3D-assisted and 125 s (range 58-248) in the conventional group.

One case-control study by Cai et al. [4], combining 3D visualization with a 3D printed model for screw placement, reported a significant decrease in fluoroscopy frequency ( $29 \pm 4$ versus $63 \pm 3 ; p<0.001$ ) compared to the conventional technique.

\subsection{Post-Operative Results}

Our second question asks about the difference in post-operative results in terms of fracture reduction and functional outcome.

\subsubsection{Screw Malposition}

Two out of five case-control studies reported significantly less screw malposition rates by using a 3D printed drilling [16] and intra-operative 3D image guided surgery [17]. Two other studies found no difference using intra-operative 3D image guided surgery $[14,18]$. Beck et al. [22] did not report on the difference expressed by a $p$-value. Weighted mean screw malposition rate for 3D-assisted surgeries was $8 \%$ (range $0-22.6$ ) compared to $18 \%$ (range 5-24) in the conventional group (varying from contacting cortical bone to actual perforation of the cortical bone).

\subsubsection{Post-Operative Reduction Score}

Two case-control studies did not report an improved quality of the reduction of the fracture by using a 3D printed model [4] or a 3D printed drilling guide [16]. Weighted mean reduction score was $86 \%$ (range 79-100) for 3D-assisted surgery and $82 \%$ (range 81-89) in the conventional group according to the Tornetta and Matta criteria [29].

\subsubsection{Functional Outcome}

The two-case control-studies did not report an increase in functional outcome using a 3D printed model [4] or intra-operative 3D imaging [17]. Weighted mean rate of the Majeed score "excellent" and "good" for 3D-assisted surgery was 88\% (range 82-100) and 83\% (range 81-89) in the conventional group. 


\subsection{Best-Evidence Synthesis}

\subsubsection{Intra-Operative Results}

Compared to conventional pelvic ring injury surgery, moderate evidence was found for a decrease in operation time per screw, operation time overall, blood loss, fluoroscopy dose and fluoroscopy time. The evidence for a decrease in fluoroscopy frequency was limited. Conflicting evidence was found for a decrease in screw malposition rate.

\subsubsection{Post-Operative Results}

Moderate evidence was found that fracture reduction as well as functional outcome did not improve using 3D assisted pelvic ring injury surgery.

\section{Discussion}

No overview exists on the currently available 3D technologies and to what extent they contribute to the operative treatment of pelvic ring injuries. In this systematic review we evaluated outcomes of the complete spectrum of innovative 3D technologies applied for pelvic ring injury surgery over the past decade. Thereby, it provides a clinically questiondriven overview about the ongoing debate whether these advanced 3D technologies contribute to the results of operations and patient recovery. It encompasses 18 articles, showing that previously applied 3D-assisted pelvic ring injury surgery can be divided in five main groups. These include ' $3 \mathrm{D}$ virtual fracture visualization and preoperative planning', '3D printed model assisted surgery', 'pre-contouring of osteosynthesis material', '3D printed surgical guides', and 'intra-operative 3D imaging'. The results reveal that the application of these technologies seem to have a positive effect on the operative treatment of pelvic ring injuries by shortening the duration of surgery, decreasing blood loss as well as fluoroscopy frequency, dose and time and minimizing risks on screw malposition. No difference in fracture reduction and functional outcome between 3D-assisted and conventional surgery was established.

Limitations of this systematic review are considered small patient groups and a wide range of methodological quality including a substantial number of moderate and poorquality studies. Hence, a best-evidence synthesis was performed which is a transparent and commonly applied method attempting to answer the key questions [31,32]. Moreover, high heterogeneity between the studies was observed in terms of different outcome variables used. As a result, a limited number of comparative studies addressed all outcome variables of interest.

Our first question concerned the effects of 3D-assisted surgery on intra-operative outcomes including operation time, blood loss, fluoroscopy time, dose and frequency as well as screw malposition. The results of this systematic review reveal some potential intra-operative advantages by using 3D-assisted surgery. Overall, operative time can be reduced by using 3D printed models. This is in line with a meta-analysis performed by Zhang et al. [33]. Additionally, operation time per screw is shown to be decreased using 3D navigation in percutaneous sacroiliac screw placement. One case-control study showed that blood loss might be reduced by using $3 \mathrm{D}$ printing assisted contoured template compared to conventional surgery [3]. Fluoroscopy time can be effectively reduced by using 3D techniques as shown by three case-control studies $[14,17,28]$. Moreover, fluoroscopy dose and frequency might be reduced, although more studies are needed to actually draw conclusions with regard to these outcome measures. The majority of the articles (13 out of 18) in our systematic review reported on use of 3D navigation for percutaneous screw placement. Based on these results, we may cautiously conclude that 3D navigation tends towards a decrease in screw malposition, although larger comparative studies are needed. This is in line with the systematic review and meta-analysis by Zwingmann et al. [10].

Our second research question concerned the effects of 3D-assisted surgery on postoperative outcomes including fracture reduction and functional outcome. According to the reduction score by Tornetta and Matta, no difference could be found by using 3D-assisted surgery in comparison with conventional surgery. However, to date reduction measure- 
ments in pelvic radiographs have not been validated and interobserver reliability has shown to be poor [34]. Moreover, no evidence for improved functional outcome was found using 3D-assisted surgery for pelvic ring injuries. Nonetheless, only a limited number of studies with different methodological quality reported on these outcome measures after iliosacral screw fixation. Hence, future high-quality comparative studies on all five 3D techniques are needed to clarify whether post-operative reduction and functional outcome may benefit from 3D-assisted surgery.

\section{Conclusions}

Overall, five different techniques of 3D-assisted surgery were identified and are currently in use for pelvic ring injury treatment. These included ' $3 \mathrm{D}$ virtual fracture visualization and preoperative planning', '3D printed model assisted surgery', 'pre-contouring of osteosynthesis material', '3D printed surgical guides', and 'intra-operative 3D imaging'. These 3D-based techniques offer additional tools to improve intra-operative efficiency in terms of operation time, blood loss, fluoroscopy dose, time and frequency as well as accuracy of screw placement. However, improved anatomical reduction or functional outcome following 3D-assisted surgery has not been established so far. Due to the heterogeneity of the included studies in terms of methodological quality and number of studies that evaluated each of the outcomes of interest, results should be interpreted with caution. Future high-quality comparative studies are necessary to further establish possible advantages of $3 \mathrm{D}$-assisted surgery in the treatment of pelvic ring injuries.

Supplementary Materials: The following are available online at https:/ / www.mdpi.com/article/ 10.3390/jpm11090930/s1, Supplemental Digital Content 1—overview of 3D assisted pelvic ring injury surgery.

Author Contributions: H.B. contributed to the conception of the work, data collection, data analysis, drafting of the article and critical revision. A.M.L.M. contributed data collection and critical revision. K.t.D. contributed to the conception of the work and critical revision. J.N.D., M.E.M. and E.H. all contributed to the critical revision of the work. I.H.F.R. contributed to the conception of the work, data analysis and critical revision. F.F.A.I. contributed to the conception of the work, data analysis and critical revision. All authors have read and agreed to the published version of the manuscript.

Funding: This research received no external funding.

Institutional Review Board Statement: Not applicable.

Informed Consent Statement: Not applicable.

Acknowledgments: We thank Truus van Ittersum for helping with the search strategy.

Conflicts of Interest: The authors declare no conflict of interest.

\section{References}

1. Pohlemann, T.; Tosounidis, G.; Bircher, M.; Giannoudis, P.; Culemann, U. The German multicentre pelvis registry: A template for an European expert network? Injury 2007, 38, 416-423. [CrossRef]

2. Verbeek, D.O.; Ponsen, K.J.; Fiocco, M.; Amodio, S.; Leenen, L.P.H.; Goslings, J.C. Pelvic fractures in the Netherlands: Epidemiology, characteristics and risk factors for in-hospital mortality in the older and younger population. Eur. J. Orthop. Surg. Traumatol. 2017, 28, 197-205. [CrossRef] [PubMed]

3. Hung, C.-C.; Li, Y.T.; Chou, Y.-C.; Chen, J.-E.; Wu, C.-C.; Shen, H.-C.; Yeh, T.-T. Conventional plate fixation method versus pre-operative virtual simulation and three-dimensional printing-assisted contoured plate fixation method in the treatment of anterior pelvic ring fracture. Int. Orthop. 2019, 43, 425-431. [CrossRef]

4. Cai, L.; Zhang, Y.; Chen, C.; Lou, Y.; Guo, X.; Wang, J. 3D printing-based minimally invasive cannulated screw treatment of unstable pelvic fracture. J. Orthop. Surg. Res. 2018, 13, 71. [CrossRef] [PubMed]

5. Gras, F.; Marintschev, I.; Wilharm, A.; Klos, K.; Mückley, T.; O Hofmann, G. 2D-fluoroscopic navigated percutaneous screw fixation of pelvic ring injuries—a case series. BMC Musculoskelet. Disord. 2010, 11, 153. [CrossRef] [PubMed]

6. Balling, H. 3D image-guided surgery for fragility fractures of the sacrum. Oper. Orthop. Traumatol. 2019, 31, 491-502. [CrossRef] [PubMed]

7. Zwingmann, J.; Konrad, G.; Kotter, E.; Südkamp, N.P.; Oberst, M. Computer-navigated iliosacral screw insertion reduces malposition rate and radiation exposure. Clin. Orthop. Relat. Res. 2009, 467, 1833-1838. [CrossRef] [PubMed] 
8. Moher, D.; Liberati, A.; Tetzlaff, J.; Altman, D.G.; PRISMA Group. Preferred reporting items for systematic reviews and meta-Analyses: The prisma Statement. PLoS Med. 2009, 6, e1000097. [CrossRef]

9. Banierink, H.; Duis, K.T.; Wendt, K.; Heineman, E.; Ijpma, F.; Reininga, I. Patient-reported physical functioning and quality of life after pelvic ring injury: A systematic review of the literature. PLoS ONE 2020, 15, e0233226. [CrossRef]

10. Zwingmann, J.; Hauschild, O.; Bode, G.; Südkamp, N.P.; Schmal, H. Malposition and revision rates of different imaging modalities for percutaneous iliosacral screw fixation following pelvic fractures: A systematic review and meta-analysis. Arch. Orthop. Trauma Surg. 2013, 133, 1257-1265. [CrossRef]

11. Pastor, T.; Tiziani, S.; Kasper, C.D.; Pape, H.-C.; Osterhoff, G. Quality of reduction correlates with clinical outcome in pelvic ring fractures. Injury 2019, 50, 1223-1226. [CrossRef]

12. Ouzzani, M.; Hammady, H.; Fedorowicz, Z.; Elmagarmid, A. Rayyan-a web and mobile app for systematic reviews. Syst. Rev. 2016, 5, 1-10. [CrossRef]

13. Law, M.; Stewart, D.; Pollock, N.; Letts, L.; Bosch, J.; Westmoreland, M. Guidelines for Critical Review Form-Quantitative Studies [Internet] Quantitative Review Form-Guidelines. 1998. Available online: https://healthsci.mcmaster.ca/docs/librariesprovider1 30/default-document-library / guidelines-for-critical-review-form-quantiative-studies-english.pdf?sfvrsn=ee9f6c19_2 (accessed on 1 July 2021).

14. Berger-Groch, J.; Lueers, M.; Rueger, J.M.; Lehmann, W.; Thiesen, D.; Kolb, J.P.; Hartel, M.J.; Grossterlinden, L.G. Accuracy of navigated and conventional iliosacral screw placement in B- and C-type pelvic ring fractures. Eur. J. Trauma Emerg. Surg. 2018, 46, 107-113. [CrossRef]

15. Takao, M.; Hamada, H.; Sakai, T.; Sugano, N. Factors influencing the accuracy of iliosacral screw insertion using 3D fluoroscopic navigation. Arch. Orthop. Trauma Surg. 2018, 139, 189-195. [CrossRef]

16. Yang, F.; Yao, S.; Chen, K.-F.; Zhu, F.-Z.; Xiong, Z.-K.; Ji, Y.-H.; Sun, T.-F.; Guo, X.-D. A novel patient-specific three-dimensionalprinted external template to guide iliosacral screw insertion: A retrospective study. BMC Musculoskelet. Disord. 2018, 19, 1-9. [CrossRef] [PubMed]

17. Li, B.; He, J.; Zhu, Z.; Zhou, N.; Hao, Z.; Wang, Y.; Li, Q. Comparison of 3D C-arm fluoroscopy and 3D image-guided navigation for minimally invasive pelvic surgery. Int. J. Comput. Assist. Radiol. Surg. 2015, 10, 1527-1534. [CrossRef] [PubMed]

18. Teo, A.Q.A.; Yik, J.H.; Keat, S.N.J.; Murphy, D.P.; O'Neill, G.K. Accuracy of sacroiliac screw placement with and without intraoperative navigation and clinical application of the sacral dysmorphism score. Injury 2018, 49, 1302-1306. [CrossRef] [PubMed]

19. Li, B.; Chen, B.; Zhang, Y.; Wang, X.; Wang, F.; Xia, H.; Yin, Q. Comparative use of the computer-aided angiography and rapid prototyping technology versus conventional imaging in the management of the tile C pelvic fractures. Int. Orthop. 2015, 40, 161-166. [CrossRef]

20. Takeba, J.; Umakoshi, K.; Kikuchi, S.; Matsumoto, H.; Annen, S.; Moriyama, N.; Nakabayashi, Y.; Sato, N.; Aibiki, M. Accuracy of screw fixation using the $\mathrm{O}$-arm ${ }^{\circledR}$ and StealthStation ${ }^{\circledR}$ navigation system for unstable pelvic ring fractures. Eur. J. Orthop. Surg. Traumatol. 2017, 28, 431-438. [CrossRef] [PubMed]

21. Pieske, O.; Landersdorfer, C.; Trumm, C.; Greiner, A.; Wallmichrath, J.; Gottschalk, O.; Rubenbauer, B. CT-guided sacroiliac percutaneous screw placement in unstable posterior pelvic ring injuries: Accuracy of screw position, injury reduction and complications in 71 patients with 136 screws. Injury 2015, 46, 333-339. [CrossRef]

22. Beck, M.; Kröber, M.; Mittlmeier, T. Intraoperative three-dimensional fluoroscopy assessment of iliosacral screws and lumbopelvic implants stabilizing fractures of the os sacrum. Arch. Orthop. Trauma Surg. 2010, 130, 1363-1369. [CrossRef]

23. Chen, K.; Yao, S.; Yang, F.; Drepaul, D.; Telemacque, D.; Zhu, F.; Zeng, L.; Xiong, Z.; Sun, T.; Guo, X. Minimally invasive screw fixation of unstable pelvic fractures using the "Blunt End" kirschner wire technique assisted by 3D printed external template. BioMed Res. Int. 2019, 2019, 1524908. [CrossRef] [PubMed]

24. Gao, H.; Luo, C.-F.; Hu, C.-F.; Zhang, C.-Q.; Zeng, B.-F. Minimally Invasive Fluoro-Navigation Screw Fixation for the Treatment of Pelvic Ring Injuries. Surg. Innov. 2011, 18, 279-284. [CrossRef] [PubMed]

25. Ghisla, S.; Napoli, F.; Lehoczky, G.; Delcogliano, M.; Habib, N.; Arigoni, M.; Filardo, G.; Candrian, C. Posterior pelvic ring fractures: Intraoperative 3D-CT guided navigation for accurate positioning of sacro-iliac screws. Orthop. Traumatol. Surg. Res. 2018, 104, 1063-1067. [CrossRef] [PubMed]

26. Kim, J.-W.; Oh, C.-W.; Lee, H.-J.; Min, W.-K.; Kyung, H.-S.; Yoon, S.-H.; Mun, J.-U.; Oh, J.-K. Percutaneous iliosacral screwing in pelvic ring injury using three-dimensional fluoroscopy. J. Orthop. Sci. 2013, 18, 87-92. [CrossRef] [PubMed]

27. Nie, W.B.; Ye, F.G.; Ma, J.L.; Yu, J.P.; Wang, M.X.; Zhang, Z.H.; Sun, F.J. Three-dimensional (3D) printing technology assisted by minimally invasive surgery for pubic rami fractures. Curr. Med. Sci. 2018, 38, 827-833. [CrossRef] [PubMed]

28. Privalov, M.; Beisemann, N.; Swartman, B.; Vetter, S.Y.; Grützner, P.A.; Franke, J.; Keil, H. First experiences with intraoperative CT in navigated sacroiliac (SI) instrumentation: An analysis of 25 cases and comparison with conventional intraoperative 2D and 3D imaging. Injury 2020. [CrossRef]

29. Tornetta, P.; Matta, J.M. Outcome of Operatively Treated Unstable Posterior Pelvic Ring Disruptions. Clin. Orthop. Relat. Res. 1996, 329, 186-193. [CrossRef]

30. AO/OTA Classification. Available online: https://www.aofoundation.org/Structure/Pages/default.aspx (accessed on 1 July 2021). 
31. van Tulder, M.; Furlan, A.; Bombardier, C.; Bouter, L. Editorial Board of the Cochrane Collaboration Back Review Group. Updated method guidelines for systematic reviews in the cochrane collaboration back review group. Spine (Phila Pa 1976) 2003, 28, 1290-1299. [CrossRef]

32. Treadwell, J.R.; Singh, S.; Talati, R.; McPheeters, M.L.; Reston, J.T. A framework for best evidence approaches can improve the transparency of systematic reviews. J. Clin. Epidemiol. 2012, 65, 1159-1162. [CrossRef]

33. Zhang, Y.D.; Wu, R.Y.; Xie, D.D.; Zhang, L.; He, Y.; Zhang, H. Effect of 3D printing technology on pelvic fractures:A meta-analysis. Zhongguo Gu Shang = China J. Orthop. Traumatol. 2018, 31, 465-471.

34. Lefaivre, K.A.; Blachut, P.A.; Starr, A.J.; Slobogean, G.P.; O’Brien, P.J. Radiographic Displacement in Pelvic Ring Disruption. J. Orthop. Trauma 2014, 28, 160-166. [CrossRef] [PubMed] 